\section{Cureus}

Received 06/28/2019

Review began 06/30/2019

Review ended 07/04/2019

Published 07/12/2019

\section{(C) Copyright 2019}

Kolli et al. This is an open access article distributed under the terms of the Creative Commons Attribution License CC-BY 3.0., which permits unrestricted use, distribution, and reproduction in any medium, provided the original author and source are credited.

\title{
Proctitis and Abdominal Aortic Aneurysm: Six Degrees of Separation
}

\author{
Sindhura Kolli ${ }^{1}$, Simcha Weissman ${ }^{2}$, Fahad Malik ${ }^{3}$, Owen Chan ${ }^{4}$, Mel A. Ona ${ }^{5}$ \\ 1. Clinical Obesity Medicine, NYU Langone Health, New York, USA 2. Internal Medicine, Hackensack \\ University Medical Center, North Bergen, USA 3. Internal Medicine, Richmond University Medical \\ Center, Staten Island, USA 4. Pathology, Pali Momi Medical Center, Affiliate of Hawai'i Pacific Health, \\ Honolulu, USA 5. Gastroenterology, Pali Momi Medical Center, Affiliate of Hawai'i Pacific Health, \\ Honolulu, USA
}

$\square$ Corresponding author: Simcha Weissman, simchaweissman@gmail.com Disclosures can be found in Additional Information at the end of the article

\section{Abstract}

Cytomegalovirus (CMV) is an aggressive virus responsible for a considerable amount of case fatalities. In the overwhelming majority of cases, this affects only the immunocompromised. Herein, we present a 76-year-old immunocompetent female who presented with gastrointestinal bleeding found to have rectal ulceration secondary to CMV infection. This manuscript aims to raise awareness of a rare cause of rectal bleeding. Hopefully, as such, our case will also prevent long-standing inflammation from persisting in patients with CMV and prevent it from contributing to cardiovascular pathology as seen in our patient.

Categories: Gastroenterology, Infectious Disease

Keywords: proctitis, cmv colitis, abdominal aortic aneurysm (aaa)

\section{Introduction}

Cytomegalovirus (CMV) is an aggressive, potentially fatal virus that predominantly affects the immunocompromised. Thus, when it presents in the immunocompetent it can often be misleading. Also, CMV is known to affect the gastrointestinal tract by inducing colonic ulcerations [1]. The appearance of ulceration in the rectum is obscure and can lead to misdiagnosis [2].

Moreover, like other chronic disease processes, the delay in diagnosis can lead to series of clinical manifestations. With inflammation in particular, the manifestations can be harsh and appear in the form of cardiovascular demise. Herein, we present an immunocompetent 76-yearold female with a rapid history of cardiovascular disease found to have CMV-induced ulcers likely responsible for her cardiovascular phenomenon via inducing a long-standing state of systemic inflammation.

\section{Case Presentation}

A 76-year-old immunocompetent female presented to the emergency department (ED) complaining of chest pain and blood in the stool. She was recently discharged from the hospital status post endovascular repair for an abdominal aortic aneurysm (AAA) and hemodialysis for worsening renovascular disease. Troponin proteins were elevated, and electrocardiogram showed a non-ST-elevation myocardial infarction (NSTEMI), for which she underwent stent placement, and began anti-coagulation therapy. The lower endoscopic evaluation revealed a three-centimeter, clean-base, ulcerated non-obstructing mass containing heaped-up mucosal 


\section{Cureus}

edges in the right/anterior portion of the rectum (Figure 1).

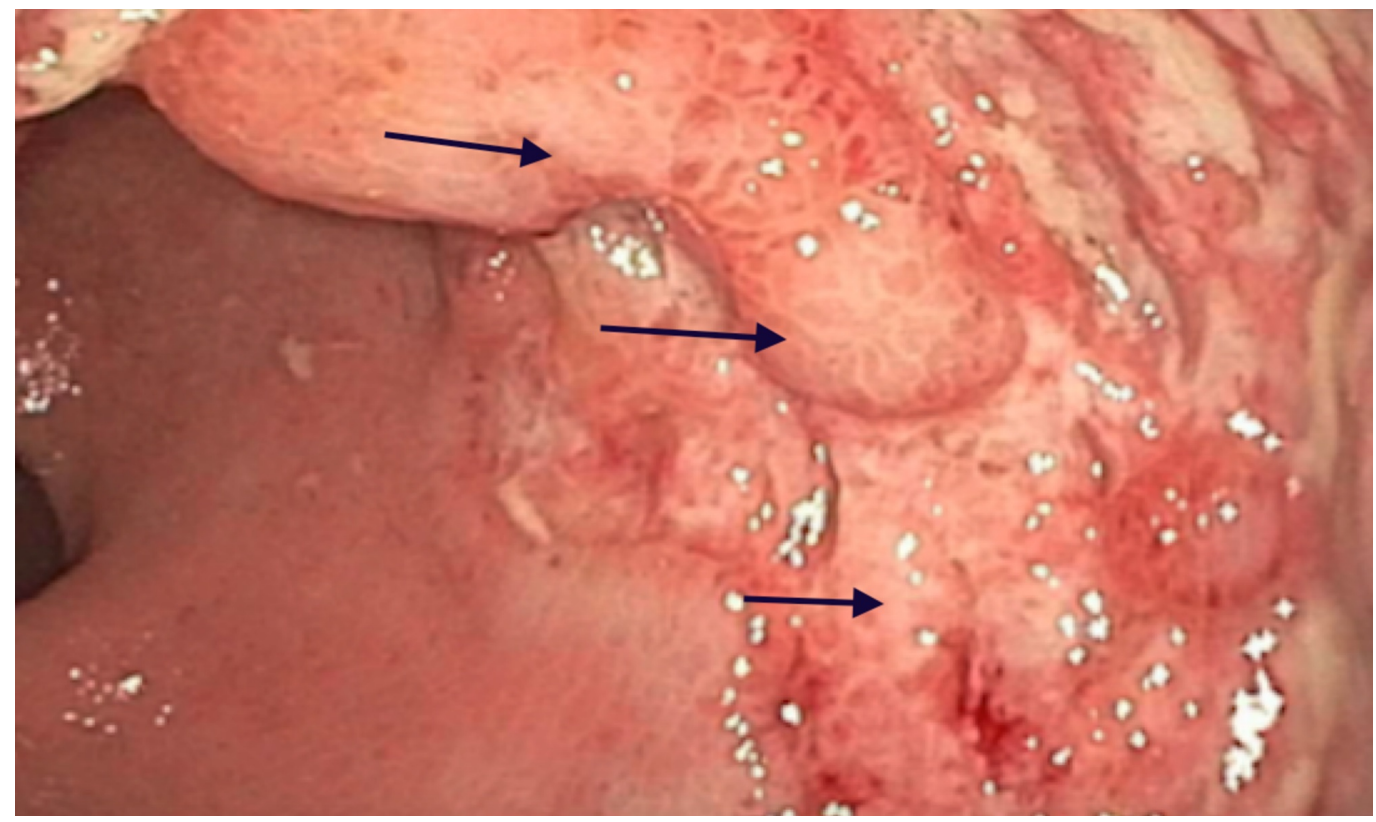

FIGURE 1: Colonoscopy revealing three centimeter $(\mathrm{cm})$ rectal ulcer with heaped-up margins (black arrows)

The differential diagnosis included tumor, inflammatory bowel disease, ischemic proctopathy, infections, and stercoral ulceration. Hematoxylin and eosin (H\&E) staining identified active proctitis with ulceration (Figure 2).

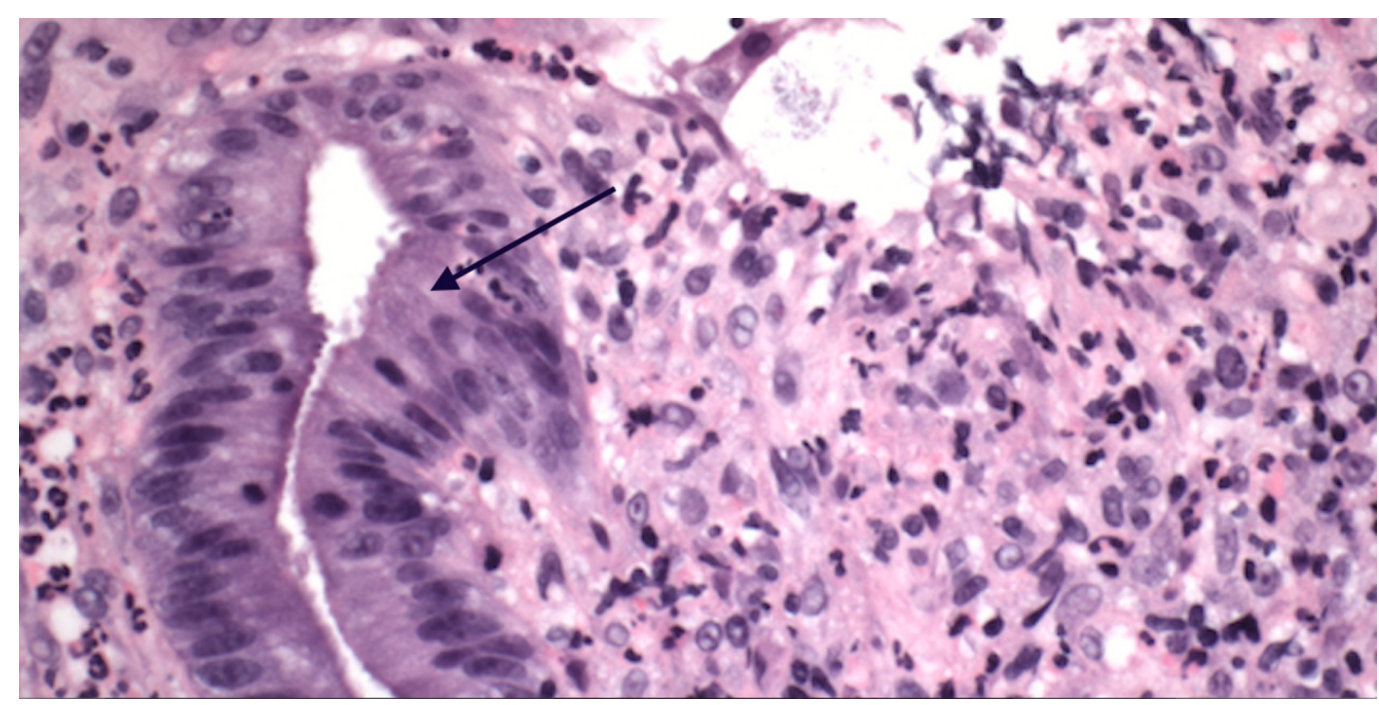

FIGURE 2: Light microscopy (hematoxylin and eosin, 400x) showing active proctitis and ulceration (black arrow)

Thereafter, immunostaining revealed CMV-infected cells, reaching a diagnosis of CMV infection without the need for seropositivity to be performed (Figure 3). 


\section{Cureus}

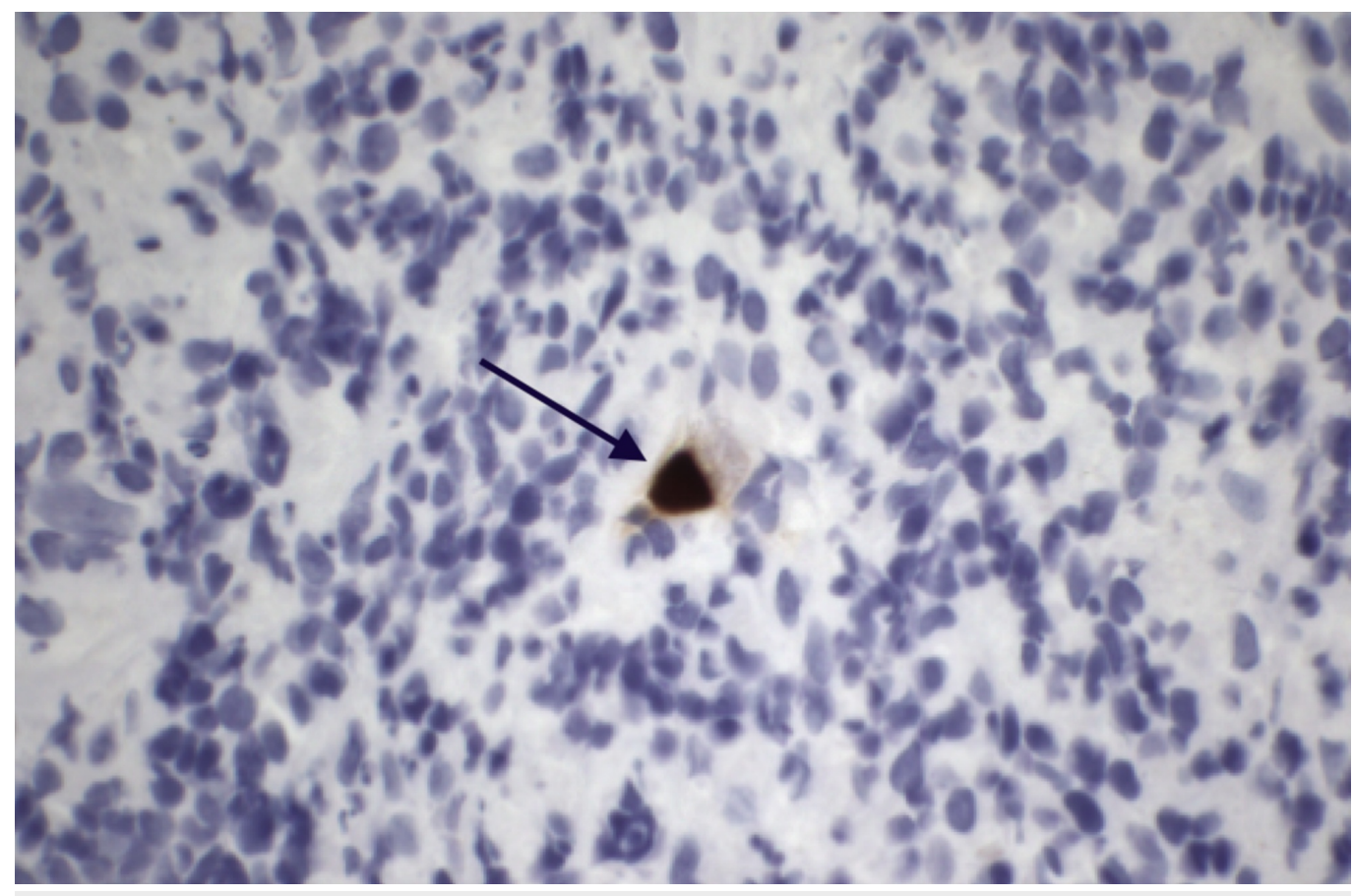

FIGURE 3: Immunostaining positive for cytomegalovirus (CMV) in the rectal ulcer with illustration of an 'owl eye inclusion' (black arrow)

She began intravenous ganciclovir and within a few days she had resolution of symptoms. She was discharged in stable condition with instructions to follow up outpatient.

\section{Discussion}

CMV-a double-stranded deoxyribonucleic acid (DNA) virus-is a prominent member of the herpes virus family. The prevalence of CMV infection in the general adult population is approximately $70 \%[3,4]$. CMV occurs via the excretion of bodily fluids and is transmission by close personal contact [5].

In the large majority of hosts, infection is usually asymptomatic [4]. Thus, usually only in the immunocompromised patient do the dangerous disease manifestations of CMV occur. CMV infection has a predilection for the lower gastrointestinal tract and primarily manifests as ulcerations along the colonic mucosa rarely involving the rectal region [3]. Our case illustrates the need to include CMV in the differential amongst the immunocompetent population especially in patients with comorbidities such as advanced kidney disease. Moreover, our case shows that ulcerations may reach as far down as the rectum and possibly contribute to disease throughout the intestine.

Attention to diagnostic clues, such as ulcers with heaped-up margins, and extra measures to immunostain a biopsied sample to search for CMV-infected cells or 'owl eye' inclusion bodies on histology, can clinch the diagnosis. This becomes of upmost importance in unsuspected patients, those chronically infected with CMV, in order to prevent systemic inflammation reactions which may ultimately lead to serious cardiovascular sequelae as seen in our case. Once diagnosed, treatment involves anti-retroviral therapy with 2-3 weeks of intravenous ganciclovir. 
Antiviral therapy is recommended for both immunocompromised and immunocompetent patients, as without this therapy CMV proctitis can otherwise result in poor patient outcomes $[4,6]$. Additionally, it is important for clinicians to become knowledgeable as to the potential adverse reactions ganciclovir treatment can induce which include myelosuppression, hepatotoxicity, and nephrotoxicity $[4,5]$.

\section{Conclusions}

In conclusion, clinicians must maintain a broad differential diagnosis in patients presenting with rectal ulcers of unknown identity. Attention to imaging and obtaining a strong patient history can help prevent fatal extra-disease sequelae. Although generally a disease of the immunocompromised, CMV can exist in the immunocompetent patient as illustrated in our patient.

\section{Additional Information \\ Disclosures}

Human subjects: Consent was obtained by all participants in this study. Conflicts of interest: In compliance with the ICMJE uniform disclosure form, all authors declare the following: Payment/services info: All authors have declared that no financial support was received from any organization for the submitted work. Financial relationships: All authors have declared that they have no financial relationships at present or within the previous three years with any organizations that might have an interest in the submitted work. Other relationships: All authors have declared that there are no other relationships or activities that could appear to have influenced the submitted work.

\section{References}

1. Lee CY, Chen YH, Lu PL: Reactivated cytomegalovirus proctitis in an immunocompetent patient presenting as nosocomial diarrhea: a case report and literature review. BMC Infect Dis. 2017, 17:113. 10.1186/s12879-017-2218-y

2. Subbarao S, O'Sullivan A, Adesina T, Gwozdz AM, Rees J, Satta G: Cytomegalovirus proctitis mimicking rectal cancer in an immunocompetent elderly patient: a case report. BMC Res Notes. 2014, 7:799. 10.1186/1756-0500-7-799

3. Nakase H, Herfarth H: Cytomegalovirus colitis, cytomegalovirus hepatitis and systemic cytomegalovirus infection: common features and differences. Inflamm Intest Dis. 2016, 1:1523. $10.1159 / 000443198$

4. Inayat F, Hussain Q, Shafique K, Tasleem SH, Hurairah A: Cytomegalovirus colitis in immunocompetent patients. Cureus. 2016, 8:10.7759/cureus.869

5. Rafailidis PI, Mourtzoukou EG, Varbobitis IC, Falagas ME: Severe cytomegalovirus infection in apparently immunocompetent patients: a systematic review. Virol J. 2008, 5:47. 10.1186/1743-422X-5-47

6. Galiatsatos P, Shrier I, Lamoureux E, Szilagyi A: Meta-analysis of outcome of cytomegalovirus colitis in immunocompetent hosts. Dig Dis Sci. 2005, 50:609-616. 